\title{
Coaching santé: un programme pour la promotion de la santé et la prévention au cabinet du médecin de famille, élaboré par le Collège de médecine de premier recours (CMPR)
}

Ueli Grüninger a,

Ruedi Hösli ${ }^{b}$, Stefan Neunerc, Margareta Schmid ${ }^{d}$

a Dr en méd., secrétaire général du Collège de médecine de premier recours (CMPR)

b M.P.H., gestionnaire de projet coaching santé CMPR

c Dr en méd., dipl. M.P.H., responsable du groupe de travail Prévention CMPR

d Dr en méd., responsable Evaluation du coaching santé CMPR

* Par souci de lisibilité, le masculin générique est utilisé pour désigner les personnes des deux sexes.

Correspondance:

Dr Ueli Grüninger,

Secrétaire général CMPR

Landhausweg 26

CH-3007 Berne

ueli.grueninger@hin.ch

www.gesundheitscoachingkhm.ch
Par son comportement, chaque personne est en mesure d'avoir une influence décisive sur sa santé et chaque personne connaît le mieux ses besoins et ses possibilités. Mais l'écart est souvent très grand entre le désir d'un comportement favorable à la santé et la réalité. Dans ce contexte, les médecins sont appelés à jouer un rôle important sur la base de la confiance qui leur est témoignée, du caractère fiable de leurs prestations et de leur position dans la société.

\section{Introduction}

La promotion de la santé et la prévention sont parmi les tâches quotidiennes les plus importantes du médecin de famille lorsqu'il donne ses consultations.

Le projet coaching santé du Collège de médecine de premier recours (CMPR) a pour but de faciliter la prévention et la promotion de la santé lors des consultations en donnant accès à deux défis souvent négligés d'une intervention qui se veut efficace:

- Intégration durable du patient avec ses motivations et idées personnelles aux fins de mobiliser les compétences et ressources individuelles en matière de santé.

- Manière de procéder lors de constellations de risques variables d'un individu à l'autre et lors de comportements à risque souvent co-existants.

A cet effet, le CMPR a développé le nouveau projet «Coaching santé»; elle le teste actuellement dans le cadre d'un projet pilote déployé dans le canton de St-Gall. Ce projet vise les comportements à risque les plus fréquents et s'en occupe dans le cadre d'un programme modulaire commun pour la promotion de la santé et la prévention au cabinet médical. Le partenariat déjà existant entre patient* et médecin est mis à profit, la collaboration a lieu aussi bien lors des consultations que dans la vie quotidienne du patient.

\section{Nouvelle distribution des rôles}

Dans le coaching santé, le patient obtient le rôle principal. Il développe activement son projet santé person- nel et l'applique de manière autonome tout en demandant le soutien nécessaire qui lui convient en collaboration avec son médecin, au lieu de rester dans le rôle passif de la personne à soigner.

Le médecin devient ainsi le coach du patient. Il l'encourage et mobilise ses compétences, ses ressources et sa responsabilité personnelle en matière de santé au lieu de lui donner des conseils directifs et des recettes paternalistes.

Le médecin et le patient forment une équipe et utilisent les offres et les ressources externes de la commune de domicile du patient.

Conjointement avec son médecin de famille, le patient examine sa situation, ses objectifs et le chemin pour y parvenir:

- Est-ce que j'aimerais faire quelque chose pour ma santé?

- Quel est mon comportement actuel?

- Où et comment puis-je améliorer durablement quelques aspects de mon comportement?

- Comment vais-je fixer mes objectifs conjointement avec mon médecin de famille?

Le patient développe son propre projet santé et le met lui-même en œuvre. Le médecin de famille l'accompagne en qualité de coach sur le chemin qui mène au but visé.

\section{Processus individuel et cyclique du changement de comportement}

Le coaching santé tient compte du fait que les patients viennent avec des idées et des modèles de comportement très différents en fonction de leur parcours et de leur personnalité. Le déroulement du conseil au cabinet médical est construit sur la nature cyclique du changement des idées et des comportements en matière de santé. Cette démarche en quatre phases progressives «Sensibiliser-Motiver-Planifier-Accompagner» permet au médecin de procéder par étapes au cabinet médical selon la personnalité du patient. Elle lui permet en outre de prévoir ces étapes de manière différenciée et de les aborder plus ou moins rapidement en fonction du besoin de changement et de la disponibilité du 
patient. Cette manière de procéder est une opérationnalisation du modèle transthéorique (MTT) pour le conseil médical en vue du changement des comportements à risque.

Chaque phase du coaching santé est une étape d'apprentissage pour le patient (cf. ill. 1):

- Phase 1 - Sensibiliser: aider le patient à passer de la précontemplation à la contemplation.

- Phase 2 - Motiver: aider le patient à passer de la contemplation à la préparation.

- Phase 3 - Planifier: aider le patient à passer de la préparation à l'action.

- Phase 4 -Accompagner: aider le patient à passer de l'action à la maintenance.

\section{Learning by doing - \\ le coaching santé ne connaît pas l'abandon}

Etape par étape, le coaching santé accompagne l'équipe patient/médecin et la mène par de simples algorithmes du général au particulier, tout d'abord par la sensibilisation et la motivation, puis par la sélection et la détermination d'un comportement-cible différent enfin par la planification et la mise en œuvre d'un projet santé personnel (cf. ill. 2 ci-après). Ce processus par étapes favorise les succès et réduit les échecs, ce qui facilite la mise à jour ultérieure du conseil (cf. ill. 1). De cette façon, il n'y a pas d'abandon ni de décrochage, chaque étape est importante pour une phase de changement! Il est tout à fait normal, et c'est même la règle, que certaines étapes doivent être répétées plusieurs fois au sens d'un «learning on the job».

\section{Le déroulement du coaching santé au cabinet médical}

Le coaching santé dirigé par le médecin de famille avance pas à pas sur deux rails (ill. 2). Lors de chaque consultation, le médecin et le patient préparent l'étape suivante du changement de comportement. Dans la vie quotidienne, le patient applique ce qui a été convenu et lors de la prochaine consultation, le patient et le médecin utilisent les expériences faites pour préparer l'étape suivante.

Le déroulement du conseil en coaching santé est opérationnalisé pas à pas dans un manuel destiné au médecin, avec les sujets et les questions clés pour chacune des quatre phases du conseil. Dans ce manuel, le médecin obtient des informations détaillées sur la manière de procéder en ce qui concerne la communication et son contenu. En participant à des cours, il exerce les aptitudes nécessaires sur le plan pratique.

Une feuille de travail de collaboration médecinpatient est prévue pour chacune des quatre phases. Ces feuilles guident l'équipe comme un fil rouge à travers les processus, raison pour laquelle elles sont nommées «Fil rouge», 1 à 4 (ill. 2, FR 1-4).

\section{Préparation des médecins concernés, soutien et indemnisation?}

Les médecins de famille peuvent se préparer au coaching santé sur la base de leurs riches expériences professionnelles. Néanmoins, le passage de l'exercice habituel de la médecine à de nouvelles formes de collaboration avec le patient sous forme de coaching est, pour la plupart des médecins, un défi à relever. Ils doivent, en effet, modifier leur façon de travailler suite à la nouvelle répartition des tâches, rôles et responsabilités.

La préparation à la mise en œuvre du projet coaching santé et le soutien nécessaire ont lieu de façon modulaire et en plusieurs étapes (ill. 3).

En participant à un cours avec des patients standardisés (acteurs au bénéfice d'un entraînement spécial en communication), les médecins acquièrent l'exercice pratique et concret du conseil. Cette phase constitue l'élément majeur de la préparation. Pour préparer ce cours, nous proposons une auto-évaluation sur la base de laquelle chaque médecin concerné pourra identifier ses besoins spécifiques de formation et les couvrir lui-même, outre l'entraînement en présence (matériel mis à disposition, y compris site internet «www.gesundheitscoaching-khm.ch»). De surcroît, un membre de l'équipe de projet rend visite, durant la phase de préparation, à chaque médecin concerné afin d'examiner l'intégration du projet dans l'organisation du cabinet médical ainsi que d'autres questions pratiques.

Les cercles de qualité et les visites sur place servent de soutien et d'entraînement concret. Pour rafraîchir

Illustration 1

Les quatre étapes du conseil sont axées sur les quatre phases du changement de comportement.

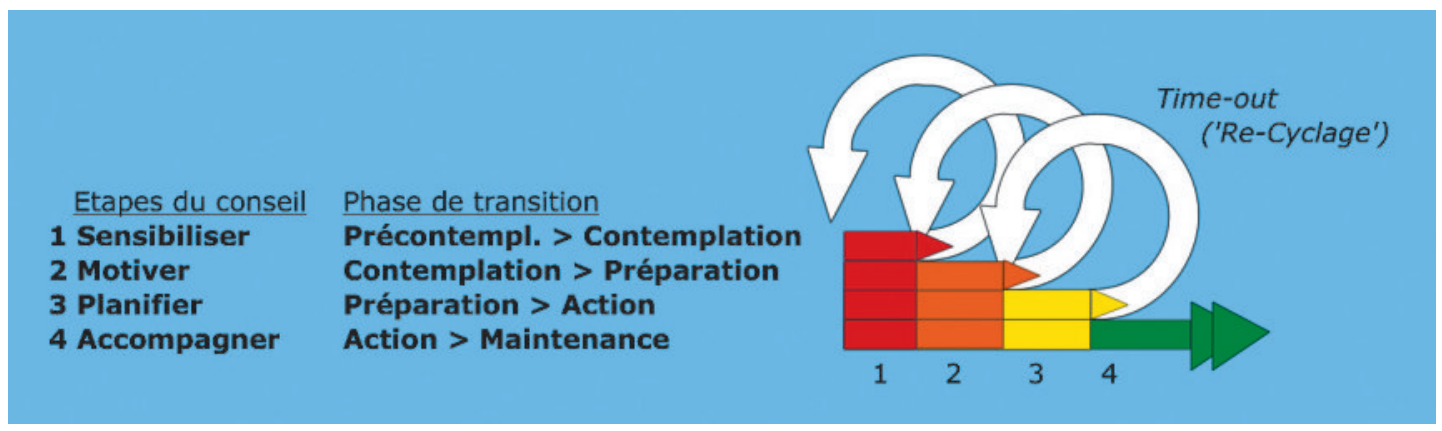


les connaissances, un jour de cours supplémentaire est également organisé avec des exercices sur des séquences avec des patients standardisés. Les médecins concernés bénéficient également d'un soutien personnalisé sous forme de supervision par vidéo et d'assistance téléphonique. En outre, un matériel pour le patient est à la disposition de chaque cabinet médical participant au projet (papillons, brochure, écrans vidéo pour la salle d'attente avec des séquences établies spécialement pour le coaching santé).

Sur la partie du site internet «gesundheitscoachingkhm.ch» sécurisée par un mot de passe, les médecins impliqués ont accès à des documents scientifiques et de cours, au matériel prévu pour le cabinet médical ainsi qu'à des liens internet et à un forum de discussion. La partie du site «www.gesundheitscoaching-

\section{Illustration 2}

Le Fil rouge, ou le déroulement du coaching santé:

- Au cours de la consultation, le médecin et le patient font un pas en direction du changement souhaité.

- Dans la vie quotidienne, le patient assimile ce qui a été fixé au cours de la consultation, il I'applique pas à pas et se prépare au prochain entretien avec son coach.

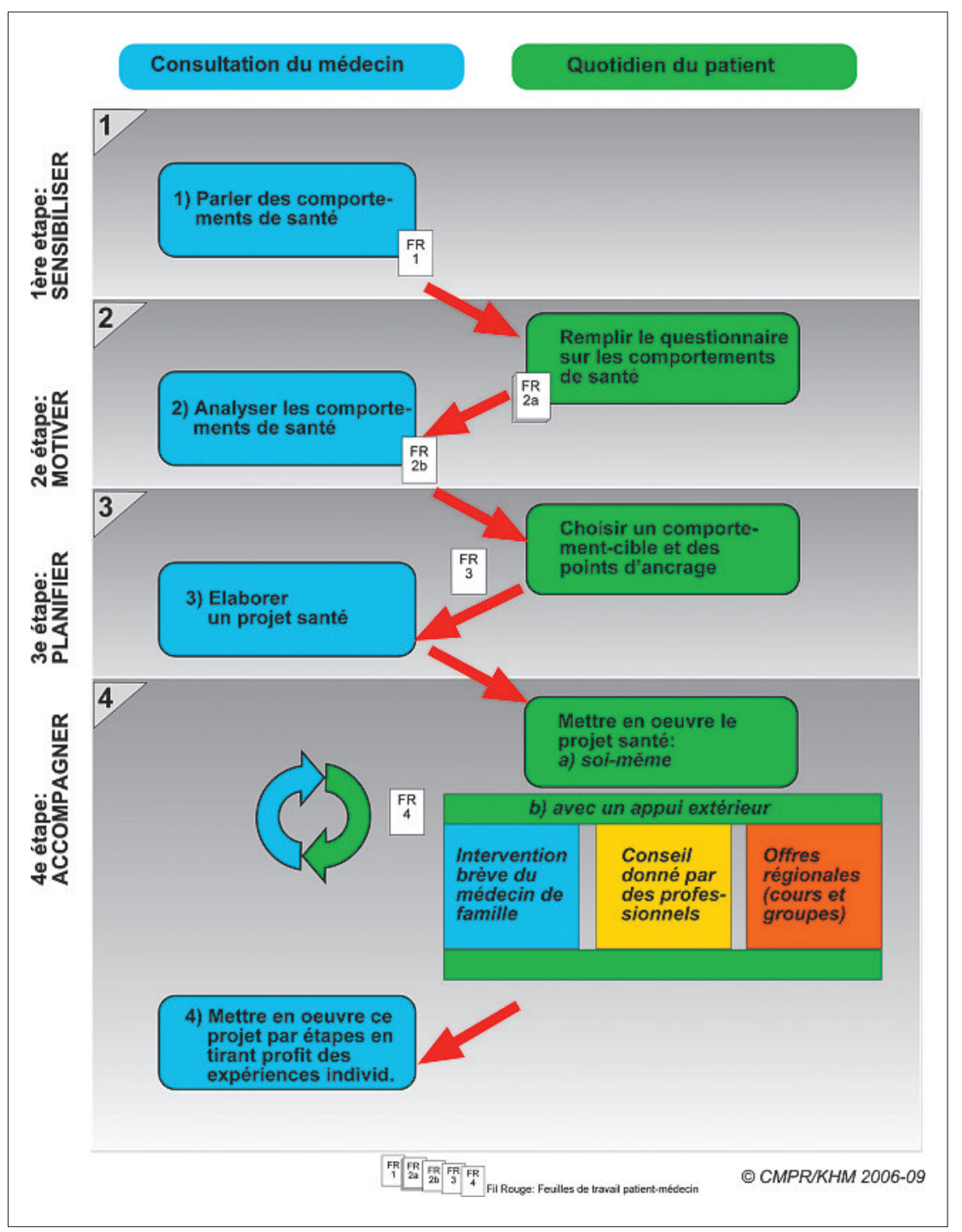

khm.ch» accessible au public donne des informations sur le coaching santé en général et sur le projet pilote dans le canton de St-Gall. Les patients trouvent là aussi des listes avec des adresses de contact pour un soutien adéquat offert par des tiers.

Le coaching donné par les médecins de famille participant au projetpilote actuel est rémunéré par des fonds propres (forfait par patient d'après le nombre de phases de changement effectuées). Il n'occasionne donc aucun frais pour les caisses-maladie et les patients, ces derniers engageant en contrepartie leur personne et leur temps. La garantie d'une rémunération adéquate en cas d'extension du projet au niveau national est l'un des thèmes de l'évaluation qui accompagne le projet pilote en cours. L'OFSP et santésuisse considèrent que le coaching santé est pour l'instant une nouvelle prestation lancée sur le marché par les médecins à titre d'entrepreneurs indépendants et qu'il leur incombe d'en attester les critères EAE. Pour cette raison, il leur est refusé de facturer leur activité de coaching par les positions tarifaires à disposition des médecins de premier recours pour leurs activités de conseil.

\section{Test pratique avant une extension au niveau national}

Le paquet initial du coaching santé a été conçu d'après son influence potentielle sur les maladies les plus répandues (burden of disease); il contient donc l'alcoolisme, le tabagisme, le manque de mouvements, l'embonpoint, l'alimentation et le stress. Ce paquet pourra être élargi à d'autres aspects et comportements importants pour la santé grâce à un cadre et à des consignes communes sur la manière de procéder.

Les responsables du projet ont achevé la phase de développement. Ils testent actuellement sa mise en œuvre, ses effets et ses coûts à échelle réelle pendant une année dans le cadre d'un vaste projet pilote avec plus de 4000 patients répartis dans 20 cabinets médicaux avant de l'étendre au niveau suisse. La phase pilote a débuté en décembre 2008 dans le canton de St-Gall en collaboration avec la société cantonale de médecine saint-galloise. Au printemps 2009, 22 cabinets médicaux ont été recrutés pour le projet. Entretemps, l'auto-évaluation, la première visite au cabinet médical et les cours ont déjà eu lieu. Les médecins de famille impliqués commenceront prochainement leur travail de coach lors des consultations.

Forts des résultats obtenus et des expériences faites avec le projet pilote, les responsables étendront ensuite le programme coaching santé aux différentes régions linguistiques de notre pays. Le but principal du projet pilote est de collecter des expériences pour optimiser les contenus, les méthodes et les processus en vue de son extension géographique. Un programme d'évaluation détaillée évalue à cette fin les données formatives et sommatives ainsi collectées.

Voici les futures lignes de développement envisageables (les premières demandes de coopération sont déjà parvenues aux responsables): 
- Elargissement du contenu: passer des six thèmes/ comportements néfastes dans le paquet initial à d'autres modules de la promotion de la santé et de la prévention.

- Elargissement thématique: application au-delà de la promotion de la santé/prévention aussi aux domaines cliniques et thérapeutiques de la médecine (p.ex. maladies chroniques, santé mentale, gestion des maladies de façon générale).

- Elargissement à d'autres consultations: p. ex. dans le domaine hospitalier (soins aigus, réadaptation, etc.).

- Elargissement à d'autres groupes-cibles: p. ex. personnes issues de l'émigration.

\section{Rôle stratégique et politique du projetpilote coaching santé}

L'objectif à long terme est l'établissement durable de la promotion de la santé et de la prévention au cabinet médical et dans la santé publique en général, de même que la création de conditions opérationnelles, stratégiques et tarifaires avantageuses.

Eu égard au système et à la politique de santé en Suisse, le projet coaching santé du Collège de Méde- cine de Premier Recours donne aussi un signal, car il prouve une fois de plus que les médecins de famille suisses s'engagent activement en faveur de la santé de la population.

\section{Financement et soutien pour le projet}

Le 31 décembre 2008, le CMPR a assuré que le projet était financé à $100 \%$ par des tiers, grâce aux contributions fédérales et cantonales et aux dons de fondations d'utilité publique et de l'économie privée, à savoir Promotion Santé Suisse (qui a aussi soutenu la phase de préparation), le Fonds national de prévention du tabagisme, Pfizer SA, Oncosuisse, l'Office fédéral de la santé publique, la Conférence des directeurs cantonaux de la santé, la Fondation HelvetiaSana, Novartis Suisse, MSD Suisse, Vivre sans tabac, Sanofi-Aventis et la Fondation suisse de cardiologie.

Cet article est le premier volet d'une série sur le thème de la promotion de la santé et de la prévention dans les cabinets médicaux.

Illustration 3

Formation continue, entrainement et support du projet coaching santé (@ KHM).

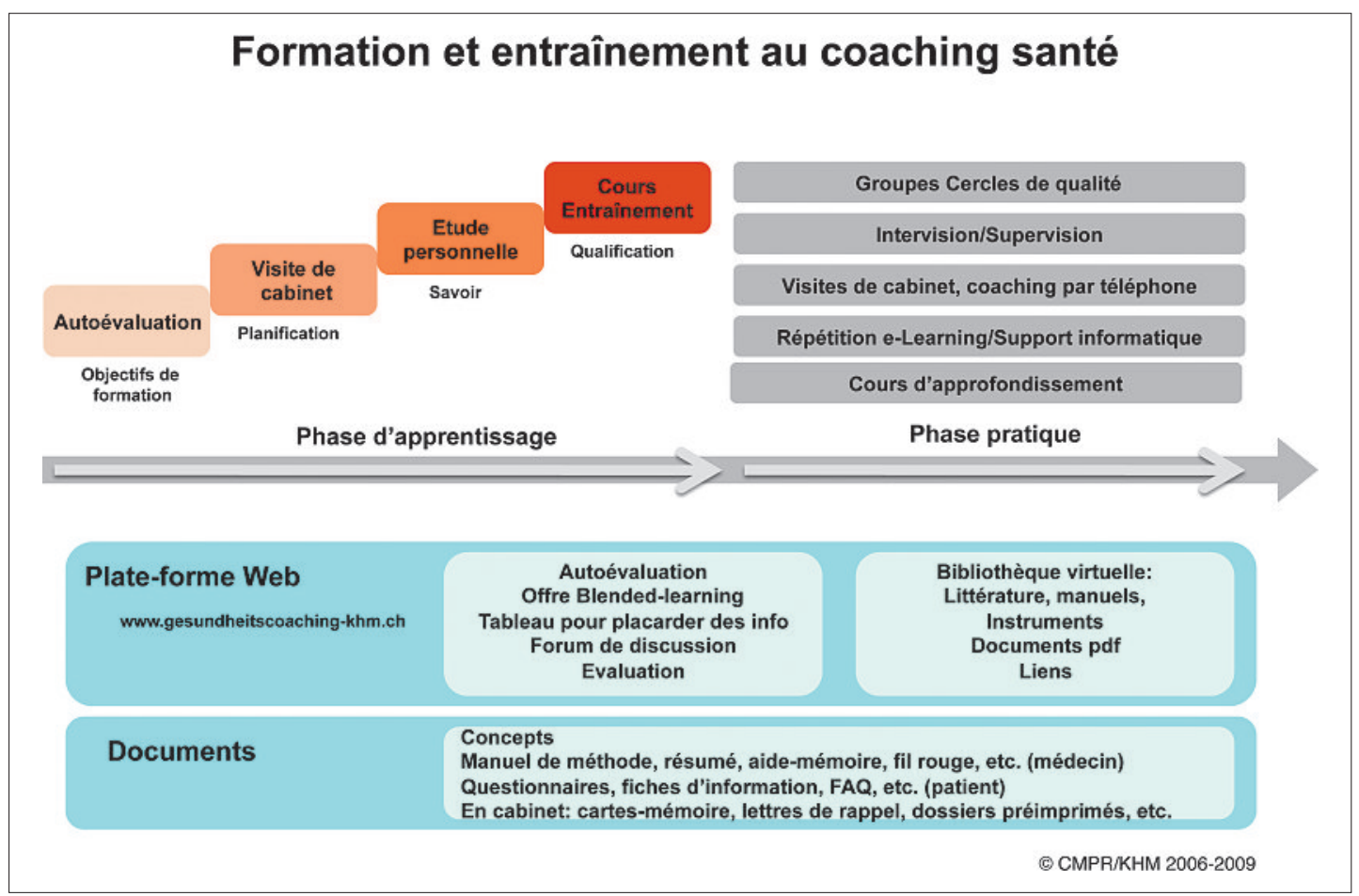

